\title{
Metodología para la fabricación de una prótesis transtibial a base de material compuesto de fibra de carbono y resina epóxica
}

\author{
Methodology for the manufacture of a \\ transtibial prosthesis based on carbon \\ fiber composite and epoxy resin
}

Miriam Siqueiros Hernández ${ }^{1}$, Marco Antonio Reyna Carranza ${ }^{2}$, Víctor Nuño ${ }^{3}$, Joel Carlos Huegel West ${ }^{4}$, Ana María Castañeda ${ }^{5}$

\footnotetext{
${ }^{1}$ Escuela de la Ciencia de la Ingeniería y Tecnología (ECITEC). Universidad Autónoma de Baja California (UABC). Blvd. Universitario \#1000, Unidad Valle de las Palmas, Tijuana, Baja California, México.

${ }^{2}$ Instituto de Ingeniería UABC Campus Mexicali. Calle de la Normal S/N y Blvd. Benito Juárez Col. Insurgentes Este Mexicali, Baja California, México.

(3,5) Universidad Autónoma de Baja California, Facultad de Ingeniería, Blvd. Benito Juárez s/n Col: Ex Ejido Coahuila, Mexicali, Baja California, México.

${ }^{4}$ Tecnológico de Monterrey. Av. Gral. Ramón Corona 2514 Col. Nvo. México Zapopan, Jal 45201, Zapopan, Jalisco, México.

e-mail: miriam.siqueiros@uabc.edu.mx ,mreyna@uabc.edu.mx; investigador.reyna@gmail.com_vnuno@uabc.edu.mx; ana.castaneda@uabc.edu.mx ,jhuegel@itesm.mx
}

\section{RESUMEN}

La problemática de las personas con capacidades diferentes y en especial de las personas amputadas, es de gran impacto en nuestra sociedad. Las prótesis actuales plantean soluciones que se alejan de las expectativas de un amputado, ya que por su elevado costo no son accesibles para la mayoría de los afectados. Esta investigación involucra la construcción de un molde para la fabricación de un prototipo de prótesis transtibial, cuyo objetivo es desarrollar una metodología de diseño, fabricación y que sea de bajo costo. Existe una gran cantidad de materiales que pueden ser utilizados en un miembro protésico, en esta experimentación se utilizó un material compuesto de fibra de carbono $3 \mathrm{~K}$ y resina epóxica, de los cuales se realizaron pruebas de tensión bajo la norma ASTM D3039 con orientación de [0/90/ \pm 45$]$ s donde se obtuvo una carga máxima de $14276,25 \mathrm{~N}$, tomando así como base los resultados obtenidos del material para llevar a cabo la fabricación del primer prototipo de prótesis transtibial el cual fue manufacturado con la técnica propuesta en esta investigación. Una vez fabricado fue sometido a pruebas de carga estática bajo el ISO 10328, donde se comprobó el laminado que se utilizó además de la metodología sugerida, se corroboró la reducción considerable en tiempo de manufactura, peso y costo promedio en relación a las prótesis comerciales.

Palabras clave: prototipo, transtibial, laminado, prótesis.

\section{ABSTRACT}

The problematic of handicap people, especially amputees, significantly impacts to the society. Existing prosthesis gives solutions that discourage amputee's expectations; high costs are not often accessible for most of them. This research includes a methodology for construction of a prototype mold for manufacturing a generic foot prosthetic, Whose objective is to develop a methodology of design, manufacture and that is of low cost. There are a large number of materials that can be used in a prosthetic limb, in this experiment a composite material of $3 \mathrm{~K}$ carbon fiber and epoxy resin was used, of which stress tests were performed under ASTM D3039 with orientation of [0/90/ \pm 45$] \mathrm{s}$ where a maximum load of 14276,25 $\mathrm{N}$ was obtained, taking as a basis the results obtained from the material to carry out the manufacture of the first prototype transtibial prosthesis which was manufactured with the technique proposed in this investigation. Once fabricated, it was subjected to static load tests under ISO 10328, where the laminate was tested, which was used in addition to 
the suggested methodology. The considerable reduction in manufacturing time, weight and average cost in relation to commercial prostheses was corroborated.

Keywords: prototype, transtibial, laminate, prosthesis.

\section{INTRODUCCIÓN}

El avance y desarrollo tecnológico de las prótesis durante los últimos años ha permitido una evolución significativa en el ámbito del diseño y de la manufactura en diversas industrias tecnológicas incluyendo la industria biomédica. Uno de los campos de utilización más importante para los materiales compuestos es en la industria ortopédica, donde su principal función es en la manufactura de prótesis.

La fabricación de un sistema protésico es un proceso en donde se involucran diferentes tipos de manufactura, que abarcan desde desarrollos artesanales hasta piezas prefabricadas. Es por ello que el proceso se debe realizar con la mejor calidad, cumpliendo así con las necesidades requeridas del paciente [1]. Para comprender la importancia de este avance tecnológico basado en el desarrollo de nuevos tipos de materiales es necesario desglosar las especificaciones tan complicadas que demandan los materiales con este tipo de aplicaciones. Entre las propiedades requeridas de los materiales compuestos para la aplicación en este sector industrial se encuentran una excelente resistencia con respecto a su peso y una biocompatibilidad superior a la de otro tipo de materiales [2]. Los materiales principales utilizados en la industria de prótesis son fibra de Vidrio, Kevlar y Carbono, por mencionar algunos. Estos materiales cuentan con propiedades mecánicas sobresalientes en comparación a los convencionales, sin embargo las ventajas que presentan entre ellos se debe a que sus características son completamente diferentes [3]. Lo pesado y voluminoso de los antiguos pies protésicos ha evolucionado tanto en diseño como manufactura, fabricándolos con materiales de alta tecnología y principalmente de fibra de carbono [4].

El presente estudio está orientado a desarrollar una metodología sistematizada para la fabricación de un prototipo de prótesis de pie para amputados transtibiales, con el objetivo de reducir el costo y tiempo de manufactura en comparación de prótesis disponibles comercialmente; además de proponer un laminado de material compuesto de fibra de carbono $3 \mathrm{~K}$ y resina epóxica, bajo la estandarización de normas para su caracterización.

\section{MATERIALES Y MÉTODOS}

\subsection{Materiales}

Para la fabricación del molde que se utilizó en la manufactura del prototipo, se emplearon barras de aluminio 6061 con tratamiento T6. El cual es una aleación estructural y sus principales aleantes son el magnesio $(\mathrm{Mg})$ y el silicio ( $\mathrm{Si}$ ). Estos metales poseen propiedades mecánicas medias, así como buenas en deformidad, maquinabilidad y gran resistencia a la corrosión. Las aleaciones del aluminio pueden endurecerse a través de tratamientos térmicos, como el T6 que consiste en aplicarle un proceso de conformado a alta temperatura.

Para garantizar el comportamiento dinámico de la prótesis de pie, se caracterizó un material compuesto, el cual se empleó fibra de carbono $3 \mathrm{~K}$ como refuerzo, este material es utilizado en prótesis debido a su capacidad de absorber energía durante su deformación, la cual es almacenada y sirve de impulso durante la marcha humana. Así mismo para la distribución de esfuerzos fue utilizada resina Epóxica System 1000 la cual actúa como un medio de distribución de esfuerzos en las fibras y protege de agentes externos, además cuenta con un curado completo a temperatura ambiente de 22 a $25^{\circ} \mathrm{C}$ con tiempos de curado de 48 a 72 horas. Las propiedades mecánicas que conforman el material compuesto utilizado se muestran a continuación (ver tabla 1).

Tabla 1: Propiedades de fibra de carbono $3 K$, y Resina Epoxica System $1000^{\circledR}$.

\begin{tabular}{l|c|c}
\hline PROPIEDADES MECÁNICAS & FIBRA DE CARBONO & RESINA EPOXICA \\
\hline Resistencia a la tracción & $3,45-4,83 \mathrm{GPa}$ & $0,294 \mathrm{Gpa}$ \\
\hline Módulo de Young & $220-241 \mathrm{GPa}$ & $18,477 \mathrm{Gpa}$ \\
\hline Elongación & $1,4-1,95 \%$ & $1,68 \%$ \\
\hline Densidad & $1,8 \mathrm{gr} / \mathrm{cm}^{3}$ & $1,13 \mathrm{gr} / \mathrm{cm}^{3}$ \\
\hline
\end{tabular}




\subsection{Métodos}

Debido a la complejidad de la geometría de las piezas desarrolladas, se eligió un procedimiento de fabricación que pudiese realizar los modelos de forma precisa y eficiente; el medio que mejor se adaptó a los requerimientos de manufactura en piezas metálicas fue el mecanizado por control numérico computarizado, con ayuda de una fresadora CNC marca HAAS ${ }^{\circledR}$ modeloVF-9 y con la implementación del software MasterCAM $^{\circledR}$ versión X5, facilitaron el maquinado de este, el cual se observa en la figura 1, donde se muestra la propuesta del molde que se utilizó y el mecanizado del mismo.

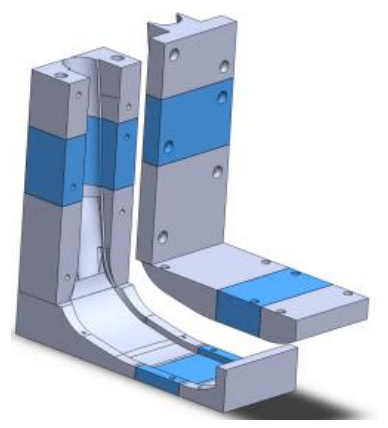

A)

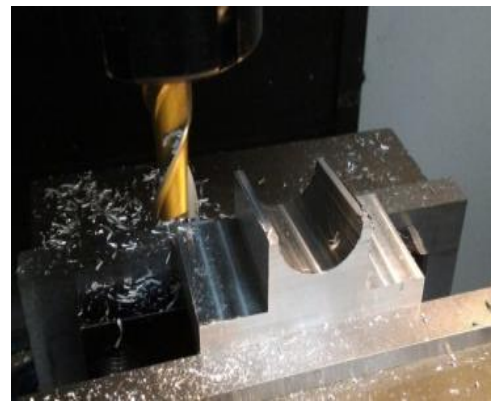

B)

Figura 1: A) Diseño de molde superior; B) Maquinado de pieza de molde

Como parte de la manufactura de diseño del molde se implementaron sujeciones mecánicas como guía de ensamble para una mejor compresión del laminado, utilizando así tornillos y pernos en diferentes secciones del molde superior e inferior, tal como se observa en la figura 2.

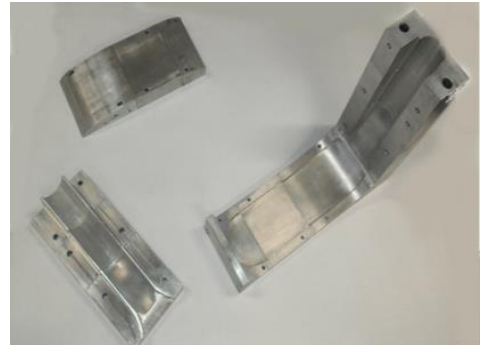

A)

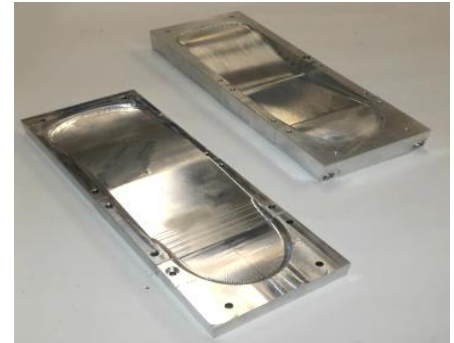

B)

Figura 2: A) Molde superior de aluminio; B) Molde inferior de aluminio

Para determinar la disposición óptima en la orientación de capas (acorde con los requerimientos mecánicos de resistencia a la tensión) y conocer el tipo de falla en el material compuesto, se contó con una fase preliminar de ensayos a tensión que fueron realizados en trabajos anteriores donde se fabricaron laminados con diferentes orientaciones de fibra $\left(0^{\circ}, 90^{\circ}, 45^{\circ}\right)$ los cuales se probaron bajo la norma ASTM D3039 [6]. De acuerdo con los resultados obtenidos la mejor combinación fue [0/90/ \pm 45$]$ s con un esfuerzo máximo de $250 \mathrm{GPa}$, en donde los esfuerzos máximos fueron distribuidos a $45^{\circ}$ sin que el material diera lugar a una fractura abrupta y a una separación completa del laminado. Por lo cual se decidió utilizar dicha combinación para la fabricación del primer prototipo.

\subsection{Elaboración del primer prototipo de prótesis transtibial.}

El prototipo se consideró como punto de partida tomando en cuenta las variables antes expuestas. El diseño se puede observar en la figura 3, la cual presenta el dimensionamiento de las dos piezas que lo conforman, indicando la cantidad y posición de los remaches utilizados. 

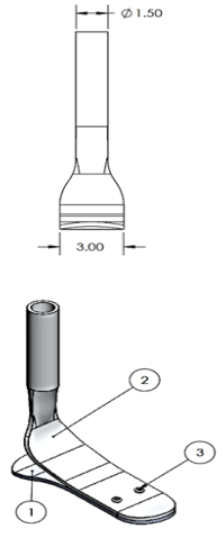
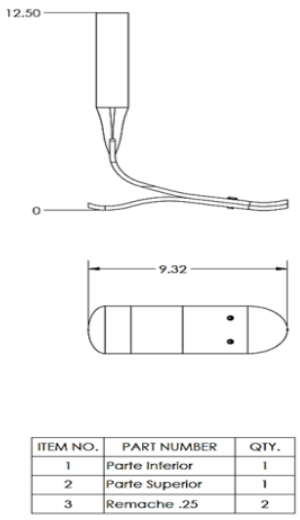

Figura 3: Plano de primer prototipo de prótesis transtibial.

El diseño utilizado fue basado en una persona de $85 \mathrm{~kg}(834 \mathrm{~N})$ y una estatura de $1,70 \mathrm{~m}$, solo como referencia para el diseño del prototipo, como se observa en la figura 4.

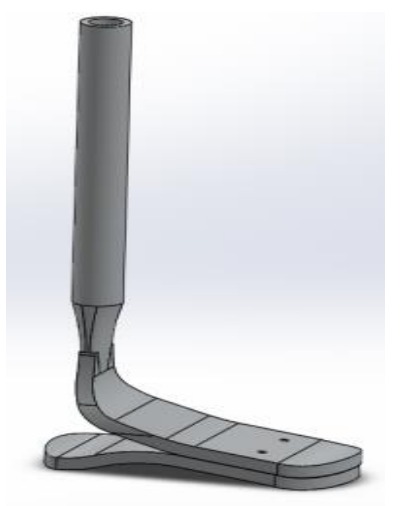

Figura 4: Diseño de prótesis transtibial.

La elaboración del primer prototipo se llevó a cabo mediante un proceso de manufactura el cual está constituido por dos etapas, la primera de ellas es la impregnación manual (HAND LAY-UP) donde se fueron colocando las diferentes capas de fibra de carbono y resina epóxica sobre el molde que se maquinó como base, y la segunda etapa fue utilizado el VACUUM BAG en donde se colocó el molde cerrado con las sujeciones mencionadas anteriormente dentro de una bolsa de vacío con la finalidad de obtener una superficie uniforme de la matriz sobre el refuerzo y así extraer los excesos de resina, para proseguir con el curado a temperatura ambiente.

El proceso de estratificación que se utilizó para elaborar el laminado simétrico fue con una orientación de [0/90/ \pm 45$]$ s; el acabado final fue un laminado a base de 8 capas. La combinación de las fibras junto con la resina finalizó con la polimerización después de haber transcurrido 72 horas de la preparación de la mezcla, a una temperatura de $25^{\circ} \mathrm{C}$.

El proceso de fabricación se realizó tomando en cuenta los siguientes pasos:

\section{a) Corte de fibra}

Se delineó la fibra con la ayuda de dos plantillas que se tomaron como guía para cada una de las piezas a realizar, el cual se procedió con el corte que se efectuó teniendo cuidado para que no se desprendieran los mechones de tela y así contar con un laminado ideal; la figura 5 muestra los cortes de las ocho capas de fibra con el direccionamiento especificado anteriormente. 


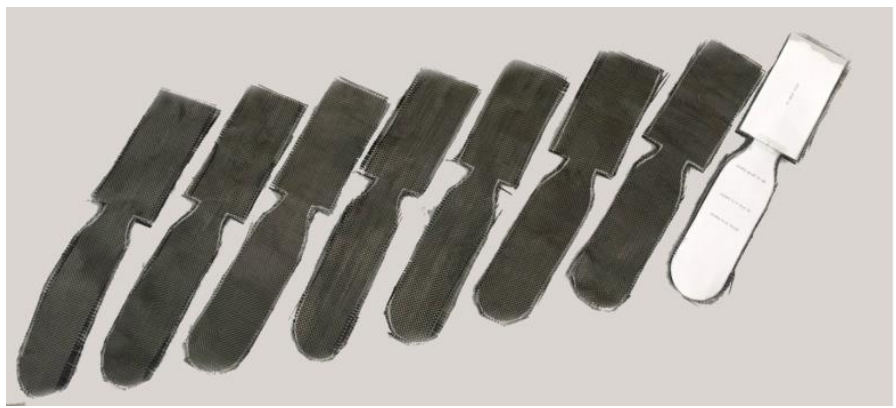

Figura 5: Fibra de carbono cortada previo a la fabricación del compuesto.

\section{b) Acondicionamiento del molde}

Para el proceso de manufactura se llevó acabo la preparación del molde de aluminio, en donde se utilizó cera como agente desmoldante, de la cual se aplicaron cinco capas dejando un lapso de 2 minutos entre la aplicación de cada una de ellas para que se adhirieran correctamente y fuera uniforme, como se observa en la siguiente figura 6.

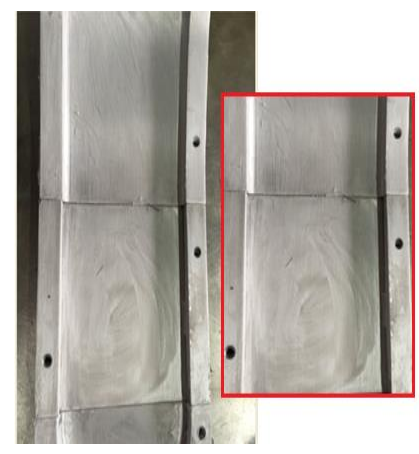

Figura 6: Aplicación de cera desmoldante al molde.

\section{c) Preparación de resina epóxica y catalizador}

Para la cantidad de resina y catalizador que se utilizó en la mezcla, se debió de tener muy presente no intentar cambiar las proporciones para no variar el tiempo de secado, debido a que su composición esta especificada por el fabricante y cualquier modificación harían que la mezcla no curara debidamente. En este estudio, el arreglo de mezclas fue de 5:1 la cual permite tener 25 minutos de trabajo máximo sin que esta tenga reacción alguna. La cantidad utilizada para la pieza superior fue de $171 \mathrm{gr}$ y para la pieza inferior fue de $120 \mathrm{gr}$, como se puede observar en la figura 7.

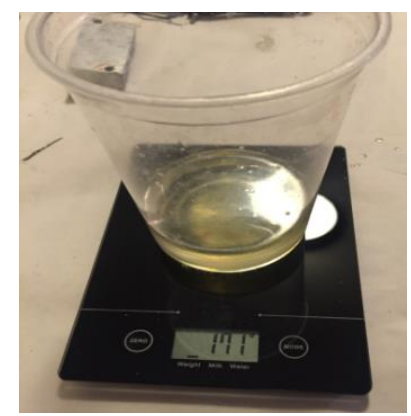

Figura 7: Preparación de resina epóxica y catalizador.

\section{d) Impregnación manual}

El método de impregnación manual (Hand lay-up) utilizado en la fabricación del prototipo consistió en colocar sobre el molde los cortes de las fibras para impregnarlos con resina epóxica por medio de una brocha 
tal como se observa en la figura 8 , posteriormente se utilizó un rodillo para la distribución adecuada de la resina y obtención de una mejor uniformidad sobre el tejido.
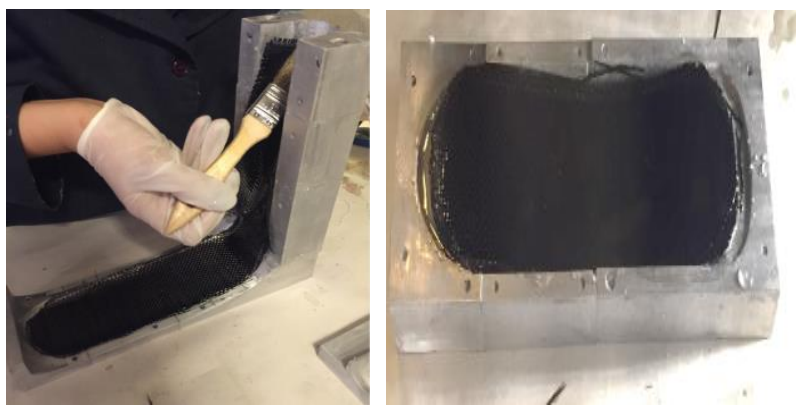

Figura 8: Distribución manual de resina.

Una vez finalizado el procedimiento de impregnación, se prosiguió con la parte del pylón que compone a la prótesis, en donde se manejó un núcleo de fibra de carbono para contar con una sección tubular del compuesto.

\section{e) Prensado de molde y método de vacío (Vacuum Bag)}

Para garantizar que el laminado quede totalmente plano y uniforme, se prosiguió a cerrar el molde iniciando con la parte superior y acoplando cada uno de los tornillos de tal forma que permaneciera cerrado por completo, para finalizar el proceso se continuo con el recubrimiento del molde con ayuda de Release Film y Breather and Bleeder los cuales ayudaron a que absorbieran los excesos de resina y así permitir colocar el molde dentro de una bolsa de vacío, la cual fue sellada por completo y con apoyo de una válvula conectada a una bomba de vacío se extrajo el aire durante un lapso de 3 minutos el cual alcanzó una presión de $-10 \mathrm{kPa}$. Para el curado de la pieza se dejó a una temperatura ambiente de $25^{\circ} \mathrm{C}$, con una humedad relativa de $20 \%$ durante 72 horas, como se muestra en la figura 9.

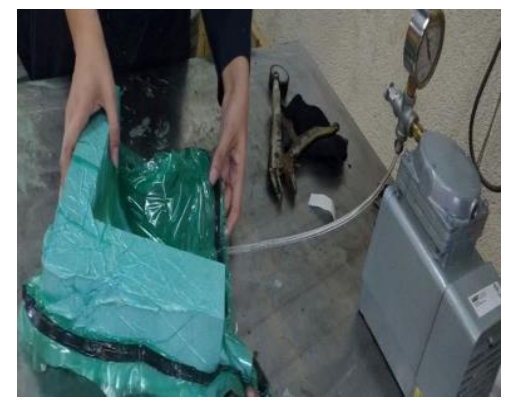

Figura 9: Molde superior sujeto a vacío.

Transcurrido este tiempo se recomienda no mover los moldes, hasta haber cumplido el tiempo estimado de curado. Posteriormente se retiró la bolsa y se inició con el destornillamiento de cada una de las piezas del molde, las cuales se realizaron con mucho cuidado para no dañar el prototipo. En la figura 10 se muestra la pieza superior e inferior separada del molde que se utilizó como base. 

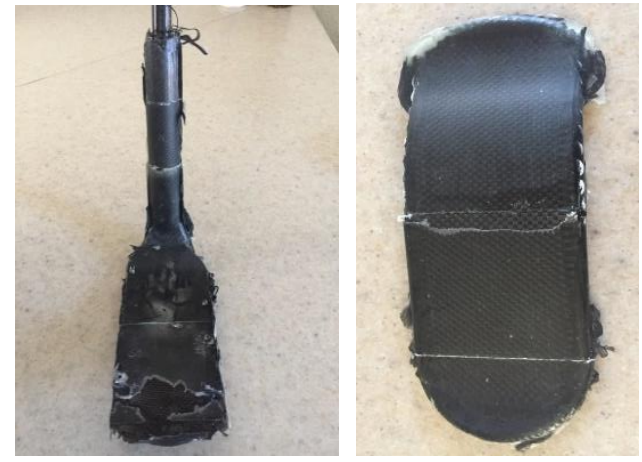

Figura 10: Prototipo de fibra de material compuesto.

\section{f) Rectificado y acabado}

En esta sección del proceso de manufactura empleado, se utilizó un dremel modelo $3000^{\circledR}$ con punta de disco y de lija para realizar el rectificado, en donde se desbastaron los excesos de fibra y resina (ver figura 11) para la obtención de un buen acabado superficial y medidas establecidas inicialmente en el diseño.
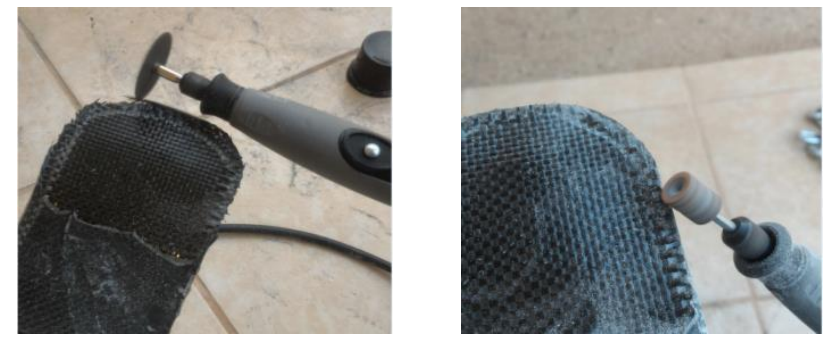

Figura 11: Prototipo de material compuesto.

La figura 12 ilustra la pieza inferior retrabajada, en la cual existe una diferencia significativa entre la superficie de la pieza desmoldada y la superficie trabajada, obteniendo así una mejora final del prototipo.

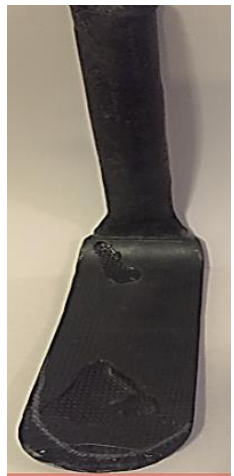

A)

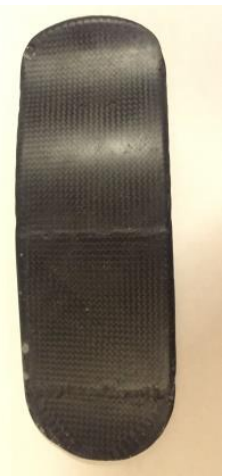

B)

Figura 12: A) Pieza superior finalizada; B) Pieza inferior finalizada.

\section{g) Unión de piezas}

Contando con las dos superficies preparadas, se prosiguió a realizar la unión con la ayuda de dos remaches modelo POP, con cuerpo de aluminio, cabeza de domo, de 3/16 pulgadas de diámetro, con agarre de 5/8 pulgadas, con mandril de acero y cabeza de ala ancha, esto con el fin de garantizar una mejor unión entre ambas piezas que conforman la prótesis, obteniendo así un espesor de laminado de $6,8 \mathrm{~mm}$ (1/4 pulgadas) por cada pieza, como se observa en la figura 13. 


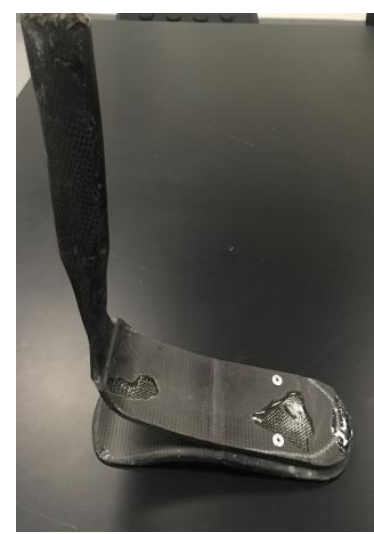

Figura 13: Prótesis unida con remaches.

\section{RESULTADOS Y DISCUSIÓN}

Los principales resultados obtenidos de los ensayos de tensión del laminado de material compuesto [0/90/ \pm 45$]$ s bajo la norma ASTM D3039 [6], se muestran en la tabla 2. Se muestran las cargas máximas presentadas en los especímenes, obteniendo el valor promedio de 14276,25 N.

Tabla 2: Resultados de carga bajo pruebas de tensión en material compuesto [0/90/ \pm 45$]$.

\begin{tabular}{c|c}
\hline \multicolumn{2}{c}{ LAMINADO [0/90/ $\mathbf{4 5}] \mathbf{s}$} \\
\hline PROBETA & CARGA (N) \\
\hline 1 & 13562,50 \\
\hline 2 & 13668,75 \\
\hline 3 & 15271,88 \\
\hline 4 & 14384,38 \\
\hline 5 & 14493,75 \\
\hline Promedio & $\mathbf{1 4 2 7 6 , 2 5 ~ N}$ \\
\hline
\end{tabular}

Respecto a las cargas obtenidas de las pruebas a tensión, se prosiguió a realizar la metodología para la fabricación del prototipo propuesto, ya que el laminado empleado, cumple con las variables establecidas del diseño. Posterior a los resultados obtenidos, se prosiguió con la obtención del molde de aluminio 6061 T6, donde se implementó el laminado mencionado en la tabla 2, para la fabricación del primer prototipo de prótesis, obteniendo así la pieza final, como se puede observar en la figura 14 .

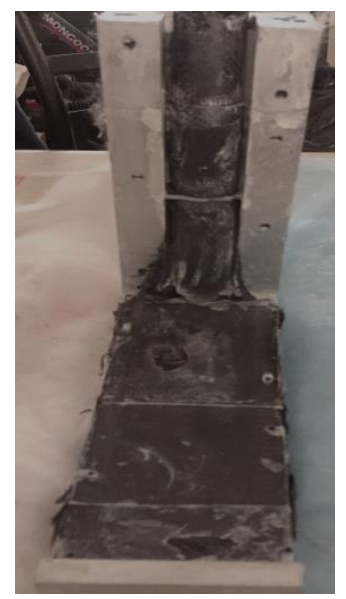

Figura 14: Pieza de material compuesto por molde de aluminio. 
Una vez desmontado el prototipo del molde se prosiguió someterlo a ensayos de compresión bajo el ISO 10328, la cual consiste en pruebas estáticas, donde identifica dos configuraciones de carga en los niveles de esfuerzo máximo presentados por las extremidades durante la marcha humana (contacto talón y contacto punta), en este estudio se basó únicamente en contacto punta.

La figura 15 muestra la pieza montada en la máquina MTS 810 Material Testing Systems con ayuda de un conector piramidal, que se utilizó como sujeción para montarla en la celda de trabajo, donde cuenta con una carga de $500 \mathrm{kN}$ y una velocidad de $0,5 \mathrm{~mm} / \mathrm{seg}$.

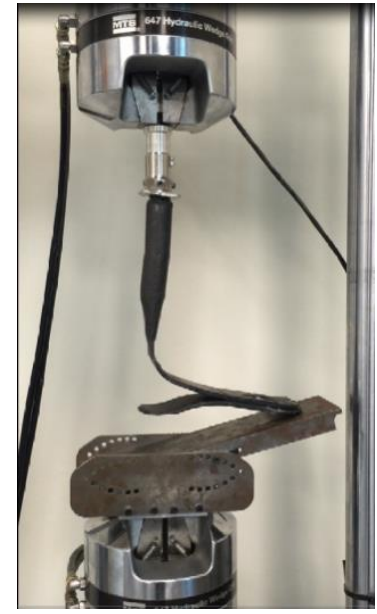

Figura 15: Sujeción de prototipo, despegue a $30^{\circ}$ de inclinación.

Cabe destacar que también se llevó a cabo la simulación de la experimentación realizada, donde se agregaron condiciones de borde que fueron necesarios para reproducir el comportamiento de la prótesis en el estado que fue ensayada. Parte de las condiciones fue aplicar una carga de $2013 \mathrm{~N}$ sobre la zona de contacto punta y restringiendo el movimiento a la altura del soporte (ver figura $16 \mathrm{~A}$ ), obteniendo así una deformación máxima de 0,2014 presentada en la sección critica del tobillo como se puede observar en la figura 16 B.

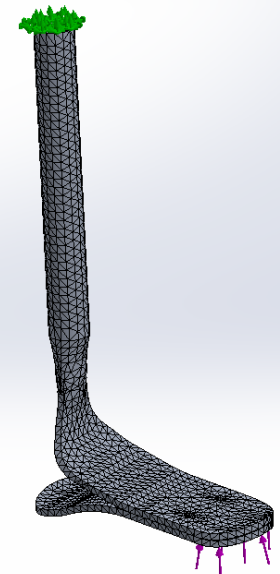

A)

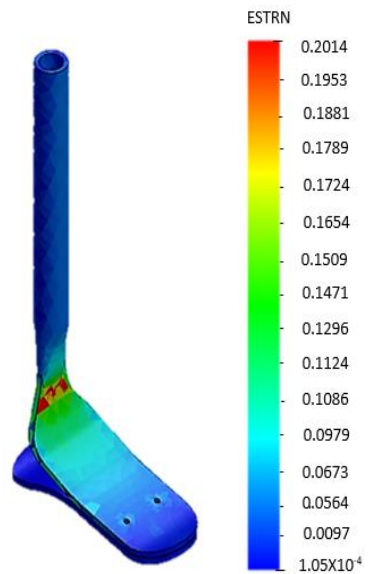

B)

Figura 16: A) Condiciones de borde para el análisis de simulación. B) Deformación presentada en la pieza.

En la figura 17 se puede observar con ayuda de un estereoscopio marca Mitutoyo ${ }^{\circledR}$ con magnificación de 40X, el daño que se presentó en la zona critica del talón, donde la matriz no presentaba suficiente refuerzo en la sección de la ruptura, esto ocasionado por la cantidad de tela de fibra de carbono que se utilizó en el laminado, lo cual es congruente con los resultados de la simulación. 


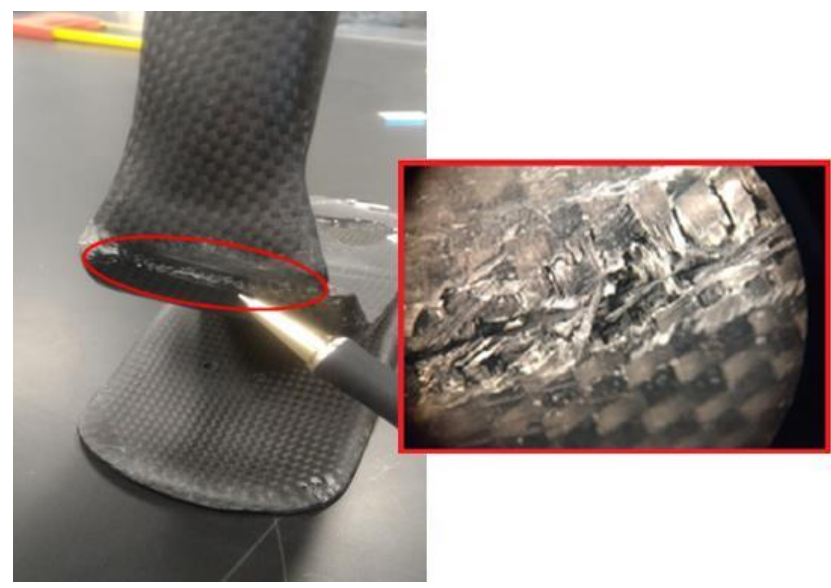

Figura 17: Fractura la sección del talón.

Con el propósito de analizar los datos establecidos en el ISO 10328, y validar los resultados obtenidos en la simulación y experimentación del prototipo, se realizó un concentrado que permitió comparar el comportamiento de las propiedades mecánicas para cada situación (ver tabla 3).

Tabla 3. Validación de datos.

\begin{tabular}{l|c|c|c}
\hline & ISO 10328 [5] & SIMULACIÓN & EXPERIMENTACIÓN \\
\hline $\mathrm{P}_{\text {máxima }}$ & $2013 \mathrm{~N}$ & $2000 \mathrm{~N}$ & $600 \mathrm{~N}$ \\
\hline Desplazamiento & $15 \mathrm{~mm}$ & $63,95 \mathrm{~mm}$ & $70 \mathrm{~mm}$ \\
\hline Puntos críticos & Talón / Punta & Punta & Punta \\
\hline
\end{tabular}

Nota: para una longitud de $317,5 \mathrm{~mm}(12,5$ pulgadas $)$

Cabe destacar el efecto de la orientación de la fibra dentro de la matriz que exhibe una variación de incremento en las propiedades mecánicas del material compuesto, al igual que en su estructura y morfología interna como se presenta a continuación.

\section{CONCLUSIÓN}

Las conclusiones de esta investigación se presentan a continuación:

Se contó con la selección de la materia prima que fue utilizada como base para realizar el arreglo del laminado propuesto, el cual fue caracterizado para conocer si era la combinación óptima que pudiese utilizar para fabricar el prototipo de prótesis transtibial. Además se diseñó y se manufacturó un molde de aluminio 6061 T6 con piezas intercambiables, mediante el proceso de maquinado en CNC, y con él poder llevar acabo la fabricación de la pieza tomando en cuenta el proceso establecido en la sección de métodos y siguiendo minuciosamente cada uno de los pasos descritos.

También gracias al equipo donde se realizó la experimentación se obtuvieron los resultados de las cargas y desplazamientos los cuales nos permitieron analizar y comparar con los tres métodos utilizados, teniendo así una mejor descripción de lo sucedido en los puntos críticos presentados.

Analizando y comparando los resultados obtenidos entre el ISO y la experimentación, se puede decir que la carga máxima presentada en el punto de ruptura fue de $600 \mathrm{~N}$ y comparada con el valor establecido, esta se mantiene a 1/3, es decir al 33\% del valor nominal estandarizado por ISO.

Por otro lado, el desplazamiento experimental fue de $70 \mathrm{~mm}$, el simulado de $63,95 \mathrm{~mm}(\varepsilon=0.2014)$ y el establecido por el ISO 10328 fue de $15 \mathrm{~mm}$, de tal manera se puede concluir que los valores de la deformación experimental y simulación, se encuentran por arriba de un $23 \%$ del valor nominal del ISO, lo cual es congruente con $33 \%$ del valor de la carga obtenida. Dicho en otras palabras, se puede decir que los resultados obtenidos pueden atribuirse principalmente a la cantidad de refuerzos o laminas que fueron empleadas. 
Es importante señalar que la sección crítica se presentó en el tobillo, haciendo de este muy poco resistente para la carga requerida, esto con un laminado de ocho capas. Sin embargo podemos asumir que el diseño del molde y el proceso de manufactura utilizado en esta investigación son favorables, ya que nos permitió analizar la orientación y la cantidad del laminado requerido para la colocación de nuevos refuerzos en los puntos críticos, dependiendo de las necesidades del sistema protésico que se tenga. Además, se pudo constatar que las condiciones de frontera que se utilizaron en la simulación, convergen con los resultados obtenidos en la experimentación.

Otro punto importante fue la comprobación del material y la combinación del laminado que se utilizó, los cuales cumplen con ciertas características favorables dentro de la pieza, es decir, con esto se puede iniciar una base de datos de materiales compuestos empleados en el área de prótesis, contando así con fichas técnicas tales como: materiales, orientaciones, combinaciones, técnicas de caracterización, etc.

\section{AGRADECIMIENTOS}

A la Universidad Autónoma de Baja California, Instituto de Ingeniería Campus Mexicali, por brindarme la oportunidad de desarrollar esta investigación; de igual manera a la Escuela de Ingeniería y Ciencias del Tecnológico de Monterrey - Campus Guadalajara por el uso del Laboratorio de Materiales donde se efectuaron algunas pruebas mecánicas.

\section{BIBLIOGRAFÍA}

[1] DALE A. BERRY, C.P. (C). "Composite Materials for Orthotics and Prosthetics", The American Orthotics and Prosthetics Associations, v. 40, n. 4, pp. 35 - 43, 1987.

[2] SCHOLZ, M.-S., BLANCHFIELD, J.P., BLOOM, L.D., et al., "The use of composite materials in modern orthopaedic medicine and prosthetic devices: A review", Composites Science and Technology, v. 71, n. 16 pp. 1791-1803, Noviembre 2011.

[3] TEOH SWEE HIL, Engineering Materials for Biomedical Applications, v. 1, Singapore, 2004.

[4] VERNON, R. R., JOHN, R. F., JOHN, W. M., et al., "Clinical Experience with Total Thermoplastic Lower Limb Prostheses", Journal of Prosthetics \& Orthotics, v. 3, pp. 51-51, 1991.

[5] ISO 10328: 2006, Prosthetics-structural testing of lower - limb prostheses - Requirements and test methods.

[6] ASTM D3039/D 3039 M-00, "Estándar Test Method for Tensile Propieties of Polymer Matrix Composite", In: ASTM INTERNATIONAL, v.08.01, ASMT Institute for Standard Resarch United States, pp. 105-116, 1997. 